\title{
Efficacy and safety of electronic cigarettes as a smoking cessation intervention: A systematic review and network meta-analysis
}

\author{
Joan M. Quigley',2, Cathal Walsh', Caitriona Lee', Jean Long', Helen Kennelly', Anne McCarthy', Paul Kavanagh ${ }^{4,5}$
}

\begin{abstract}
INTRODUCTION This systematic review of randomized controlled trials (RCTs) evaluated the efficacy and safety of electronic cigarettes (e-cigarettes, ENDS) in helping people who smoke to achieve abstinence compared with electronic non-nicotine delivery systems (ENNDS, no nicotine) or any smoking cessation comparator treatment or combination of treatments at 24-26 weeks and at 52 weeks.

METHODS Systematic review techniques involved searches of three databases in February 2020 with update searches run on 14 May 2021, two-person independent screening, two-person independent assessment of bias, formal extraction of data with verification by a second person, a feasibility assessment to decide if metaanalysis was appropriate, and network meta-analysis (NMA) of data at 24-26 weeks. Data at 52 weeks were narratively summarized.

RESULTS Ten RCTs met the inclusion criteria, eight for efficacy and ten for safety. Eight of the nine RCTs were assessed as at high risk of bias. The sample sizes of the RCTs were 30-2012. Using nicotine replacement therapy (NRT) as the reference treatment, the incidences of smoking cessation at 24-26 weeks were comparable between ENDS and NRT groups ( $R R=1.17$; 95\% CrI: 0.66-1.86). Three sensitivity analyses were carried out indicating the main findings for 24-26 weeks were robust to assumptions. The findings at 52 weeks were inconclusive. CONCLUSIONS This systematic review and NMA indicates that there is no clear evidence of a difference in effect between nicotine containing e-cigarettes and NRT on incidences of smoking cessation at 24-26 weeks, and substantial uncertainty remains.
\end{abstract}

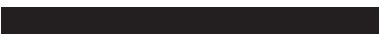

AFFILIATION

1 Health Research Board, Dublin, Ireland

2 Health Economics and Health

Technology Assessment, University of Glasgow, Glasgow, United Kingdom

3 Department of Mathematics and

Statistics, University of Limerick,

Limerick, Ireland

4 Department of Epidemiology and Public Health, Royal College of Surgeons in Ireland University of Medicine and Health Sciences, Dublin, Ireland

5 Health Intelligence Unit, Strategic

Planning and Transformation, Health Service Executive, Dublin, Ireland
\end{abstract}

\section{CORRESPONDENCE TO}

Jean Long. Health Research Board, Grattan House, 67-72 Lower Mount Street, D02 H638, Dublin, Ireland.

E-mail: jlong@hrb.ie

\section{KEYWORDS}

smoking cessation, systematic review, network meta-analysis, e-cigarettes

Received: 8 March 2021

Revised: 31 May 2021

Accepted: 14 October 2021

\section{INTRODUCTION}

An electronic cigarette (e-cigarette) is an umbrella term for an electronic device that delivers usually nicotine and/or other products, including solvents and flavorings, to the user. It is generally accepted that e-cigarettes were introduced to Europe in 2006 and to the US in 2007, and as of 2017, 433 brands of e-cigarettes and 15586 flavored e-liquids were on the market ${ }^{1}$. Although there are variations in the appearance of e-cigarettes, the National Institute on Drug Abuse has reported that regardless of their design and appearance, the devices generally operate in a similar manner and are made of similar components ${ }^{2}$. However, variations in device design and user practices mean that, as an intervention, e-cigarettes provide heterogeneous exposure to nicotine and other products delivered to the user ${ }^{3-5}$.

The emergence of e-cigarettes is a disruptive change with inter-related implications for both public health policy and day-to-day clinical 
practice $^{6-8}$. Against a context of debate on the relative harms, benefits and uncertainties ${ }^{9}$, countries are adopting shifting and diverse approaches to e-cigarette regulation in the context of their tobacco control policy ${ }^{10}$. While healthcare professionals report differing views on their role in smoking cessation for patients, some express concern about risks at individual and population level ${ }^{11}$.

Scientific evidence is essential to support both policy-makers and healthcare professions navigate and resolve difficult questions about e-cigarettes for public health and individual patient care $^{12,13}$. Systematic reviews are one critical tool in formulating recommendations in clinical guidance, and can also be mobilized to inform discussion and decision in the policy arena ${ }^{14,15}$. While the debate on e-cigarette is complex ${ }^{16}$, establishing the effectiveness of e-cigarettes as a smoking cessation aid is a central question which is especially amenable to systematic review.

Several systematic reviews have been published in this area ${ }^{17-24}$, however, the results of these reviews have been contradictory and new RCT evidence has emerged since many of these reviews were undertaken.
This systematic review evaluated the efficacy and safety of e-cigarettes in helping people who smoke to achieve abstinence at 24-26 and 52 weeks, compared with electronic non-nicotine delivery systems (ENNDS, no nicotine), or any comparator recommended for smoking cessation treatment or combination of treatments using randomized controlled trials (RCTs).

\section{METHODS}

We used systematic review techniques that involved searches of three databases (Ovid MEDLINE, Wiley Cochrane Library, and Elsevier Embase), in February 2020. These searches were updated in May 2021. The version of Embase used changed from Elsevier Embase in 2020 to Ovid Embase in 2021, which may explain the increased return from the Embase search in 2021. Controlled vocabulary was updated for each database (MeSH, EmTREE). The 2021 Ovid MEDLINE search strategy is given in the Supplementary file Appendix 1. We searched for RCTs of e-cigarettes compared with ENNDS (no nicotine), or any comparator recommended smoking cessation treatment or combination of treatments (Table 1). Our primary outcome was smoking cessation - continuous

\section{Table 1. Eligibility criteria}

\begin{tabular}{|c|c|c|}
\hline Element & Inclusion & Exclusion \\
\hline Population & Current regular smokers & $\begin{array}{l}\text { Non-smokers } \\
\text { Subgroups of smokers - pregnant women and those with } \\
\text { mental health illness } \\
\text { Dual users of combustible tobacco and e-cigarettes }\end{array}$ \\
\hline Intervention & $\begin{array}{l}\text { E-cigarette (electronic nicotine delivery system) } \pm \text { usual } \\
\text { care (i.e. smoking cessation counselling) } \\
\text { Time on treatment } \geq 6 \text { weeks }\end{array}$ & $\begin{array}{l}\text { E-cigarettes in combination with other active treatments } \\
\text { such as NRT or pharmacological interventions }\end{array}$ \\
\hline Control & $\begin{array}{l}\text { Placebo e-cigarette (without nicotine) or any comparator } \\
\text { treatment or combination of treatments usually given } \\
\text { for smoking cessation, e.g. nicotine replacement therapy }\end{array}$ & $\begin{array}{l}\text { Regular cigarettes } \\
\text { Denicotinized cigarettes }\end{array}$ \\
\hline Outcomes & $\begin{array}{l}\text { Smoking cessation: continuous abstinence measured at } 6 \\
\text { months or } 1 \text { year after treatment initiation } \\
\text { Adverse events }\end{array}$ & \\
\hline Study design & RCTs & $\begin{array}{l}\text { Observational (non-interventional) studies } \\
\text { Interventional studies with crossover design } \\
\text { Conference abstracts }\end{array}$ \\
\hline
\end{tabular}


abstinence without relapse throughout the follow-up period - as defined by European Medicines Agency guidance $^{25}$. We extracted this endpoint measured at 6 months (24-26 weeks) or 1 year (52 weeks) after treatment initiation. These endpoints are consistent with the Russell Standard on endpoints in trials of smoking cessation interventions ${ }^{26}$ and is consistent with current expert opinion ${ }^{27}$. We also extracted adverse events from identified RCTs. Completed in line with agreed systematic review principles were: two-person independent screening using the inclusion and exclusion criteria presented in Table 1; two-person independent assessment of bias using the Cochrane Risk of Bias (Version 2) tool ${ }^{28}$; formal extraction of data into a bespoke form with verification by a second person; feasibility assessment to decide if meta-analysis was appropriate following published guidance $^{29,30}$, and network meta-analysis (NMA) of data using the gemtc package in $\mathrm{R}$ version 3.6.0 where feasible.

For this analysis, we selected NRT as the reference treatment because it is a common control treatment in the RCTs identified, which is a common standard of care in international clinical guidelines for treatment of tobacco dependence ${ }^{31}$. Laboratory verified data on smoking abstinence were preferred, but self-reported data were also included.

Before the analysis was undertaken, an assessment was conducted to assess the feasibility and appropriateness of undertaking an $\mathrm{NMA}^{29,30}$. The feasibility assessment considered population, interventions and comparators, outcomes and their endpoints, and risk of bias.

Application of NMA for evidence synthesis has become widespread, and it has been used, for example, in the Cochrane review of pharmacological interventions for smoking cessation ${ }^{32}$. We used NMA to compare the multi-arm treatments at 2426 weeks in a single analysis by combining direct and indirect evidence in a single network. NMA is a next-generation evidence synthesis tool which can better serve decision-making than traditional pairwise meta-analysis through mobilizing a wider body of direct and indirect evidence across trials and improving estimate precision ${ }^{33,34}$. We used the gemtc package $^{35}$ in $\mathrm{R}$ version $3.6 .0^{36}$ to conduct an NMA of smoking cessation for e-cigarettes (electronic nicotine delivery system abbreviated to ENDS) as it allows for arm-based trial data to be analyzed and the package can summarize the comparative treatments effects as random effects relative risks. We specified the following parameters: 250000 'burn-in' iterations to be discarded, 500000 iterations for analysis, and three separate chains. Diagnostic tests were run to check model convergence. Thinning of the chains was specified to reduce the risk of autocorrelation. Default priors as specified by the gemtc package were used.

A key assumption of NMA is that of evidence consistency that is, that estimates of treatment effects from direct and indirect evidence agree. We employed the Dias et al. ${ }^{37}$ suggestion that the standard consistency model be compared with an inconsistency model. We have compared the main model (consistency model) against an inconsistency model that assumes unrelated mean (relative) effects using a function of the gemtc package $\mathrm{e}^{35}$. We also compared the direct head-to-head metaanalysis results versus the NMA outputs to further check for potential inconsistency. Meta-analyses for the direct comparisons were run using the metagen function in the meta package for the R programming language $\mathrm{l}^{37,38}$.

\section{RESULTS}

Ten RCTs reported in 16 publications $\mathrm{s}^{39-54}$ met the inclusion criteria for efficacy $(\mathrm{N}=8)^{39,40,45,47-49,51,54}$ and safety $(\mathrm{N}=10)^{39,40,45,47-49,51-54}$ of ENDS in smoking cessation. Two trials reported safety data only ${ }^{52,53}$. The PRISMA flow chart is presented in Supplementary file Appendix 4. Pertinent characteristics of the included RCTs are provided in Table 2.

The number of participants in the RCTs ranged from 30 to 2012 , with a median of 255 participants. Of the ten trials, two were based in Italy ${ }^{40,49}$, two in the $\mathrm{UK}^{45,51}$, three in the $\mathrm{USA}^{47,52,53}$ and one each in South Korea ${ }^{48}$, Canada ${ }^{54}$ and New Zealand ${ }^{39}$. Supplementary file Appendix 5 presents the detailed characteristics of the primary studies.

Smoking cessation data reported at 24-26 weeks $^{39,40,45,47-49,51,54}$ and 52 weeks ${ }^{40,45,47}$ were extracted. Supplementary file Appendix 6 presents individual RCT results for smoking cessation at 24 or 26 weeks, and at 52 weeks. When extracting data from the articles for inclusion in the systematic review and meta-analysis, self-reported data, which 
Table 2. Country, sample size, and comparators of included primary RCTs

\begin{tabular}{|c|c|c|c|c|c|c|}
\hline Study ID & $\begin{array}{c}\text { Primary } \\
\text { publication }\end{array}$ & $\begin{array}{l}\text { Secondary } \\
\text { publication }\end{array}$ & Participants & Country & Comparator 1 & Comparator 2 \\
\hline ASCEND & $\begin{array}{l}\text { Bullen et al. }{ }^{39} \\
2013\end{array}$ & - & 657 & New Zealand & $\begin{array}{l}\text { NRT monotherapy: patches } \\
(21 \mathrm{mg})\end{array}$ & $\begin{array}{l}\text { Placebo } \\
\text { e-cigarette }\end{array}$ \\
\hline ECLAT & $\begin{array}{l}\text { Caponnetto et al. }{ }^{40} \\
2013\end{array}$ & $\begin{array}{l}\text { Campagna et al. }{ }^{41} \\
2016 \\
\text { Cibella et al. }{ }^{42} \\
2016 \\
\text { Farsalinos et al. }{ }^{43} \\
2016 \\
\text { Russo et al. }{ }^{44} \\
2016\end{array}$ & 300 & Italy & Placebo e-cigarette & - \\
\hline TEC & $\begin{array}{l}\text { Hajek et al. }{ }^{45} \\
2019^{a}\end{array}$ & $\begin{array}{l}\text { Hajek et al. }{ }^{46} \\
2019^{b}\end{array}$ & 886 & UK & $\begin{array}{l}\text { NRT combination: } \\
\text { participants' choice (no } \\
\text { dose specified) }\end{array}$ & - \\
\hline $\begin{array}{l}\text { Halpern } \\
2018\end{array}$ & $\begin{array}{l}\text { Halpern et al. }{ }^{47} \\
2018\end{array}$ & - & $2012^{a}$ & USA & $\begin{array}{l}\text { No additional treatment } \\
\text { (apart from counselling) }\end{array}$ & - \\
\hline $\begin{array}{l}\text { Lee } \\
2019\end{array}$ & $\begin{array}{l}\text { Lee et al. }{ }^{48} \\
2019\end{array}$ & - & 150 & South Korea & $\begin{array}{l}\text { NRT monotherapy: gum } \\
(2 \mathrm{mg})\end{array}$ & - \\
\hline BETOFREE & $\begin{array}{l}\text { Masiero et al. }{ }^{49} \\
2019\end{array}$ & $\begin{array}{l}\text { Lucchiari et al. }{ }^{50} \\
2020\end{array}$ & 210 & Italy & Placebo e-cigarette & $\begin{array}{l}\text { No additional } \\
\text { treatment }\end{array}$ \\
\hline $\begin{array}{l}\text { Holliday } \\
2019\end{array}$ & $\begin{array}{l}\text { Holliday }{ }^{51} \\
2019\end{array}$ & - & 80 & UK & No additional treatment & - \\
\hline $\begin{array}{l}\text { Hatsukami } \\
2019\end{array}$ & $\begin{array}{l}\text { Hatsukami }^{52} \\
2019\end{array}$ & - & 152 & USA & $\begin{array}{l}\text { NRT monotherapy: gum } \\
(2 / 4 \mathrm{mg})\end{array}$ & - \\
\hline $\begin{array}{l}\text { Lee } \\
2018\end{array}$ & $\begin{array}{l}\text { Lee }^{53} \\
2018\end{array}$ & - & 30 & USA & $\begin{array}{l}\text { NRT monotherapy: patches } \\
(14 / 21 \mathrm{mg})\end{array}$ & - \\
\hline $\begin{array}{l}\text { Eisenberg } \\
2020\end{array}$ & $\begin{array}{l}\text { Eisenberg }^{55} \\
2020\end{array}$ & - & 376 & Canada & Placebo e-cigarette & $\begin{array}{l}\text { No additional } \\
\text { treatment }\end{array}$ \\
\hline
\end{tabular}

a There were 6006 participants in the five arms of the trial, but 2012 participants in the two arms included in this review.

were then biochemically verified (measuring cotinine or carbon monoxide levels), were preferred over self-reported unverified data. Biochemical verification of abstinence increases scientific rigor and is recommended in clinical trials of smoking cessation $^{55}$. Data at $24-26$ weeks were considered separately from 52 weeks data as results varied considerably between the studies that reported at both timepoints ${ }^{40,45,47}$ and there was a notable number of dropouts in all included trials across time (Supplementary file Appendix 7).

Risk of bias was assessed using the Cochrane Risk of Bias (Version 2) tool for trials ${ }^{28}$. Of the ten RCTs, nine were rated high risk of bias ${ }^{39,40,47-49,51-}$ ${ }^{54}$ and one was rated as having some concerns ${ }^{45}$.
These ratings were mainly driven by loss to followup and missing outcome data. The numbers lost to follow-up were high in all RCTs, and the proportions of successful cessation events were low in the RCTs (Supplementary file Appendix 7), both of which introduced uncertainty to the results of this systematic review. Participants who were lost to follow-up were treated as treatment failure in the RCTs and in this analysis. The number lost to follow-up was greater than $20 \%$ at 6 months in all trials, except one ${ }^{48}$, and was up to $30.3 \%$ in the study with the highest dropout rate ${ }^{52}$. Eight of the RCTs reported the numbers lost to follow-up, seven of these reported it separately by arm, and in six trials the number lost-to-follow-up was highest in the NRT 
Figure 1. Risk of bias in ENDS trials

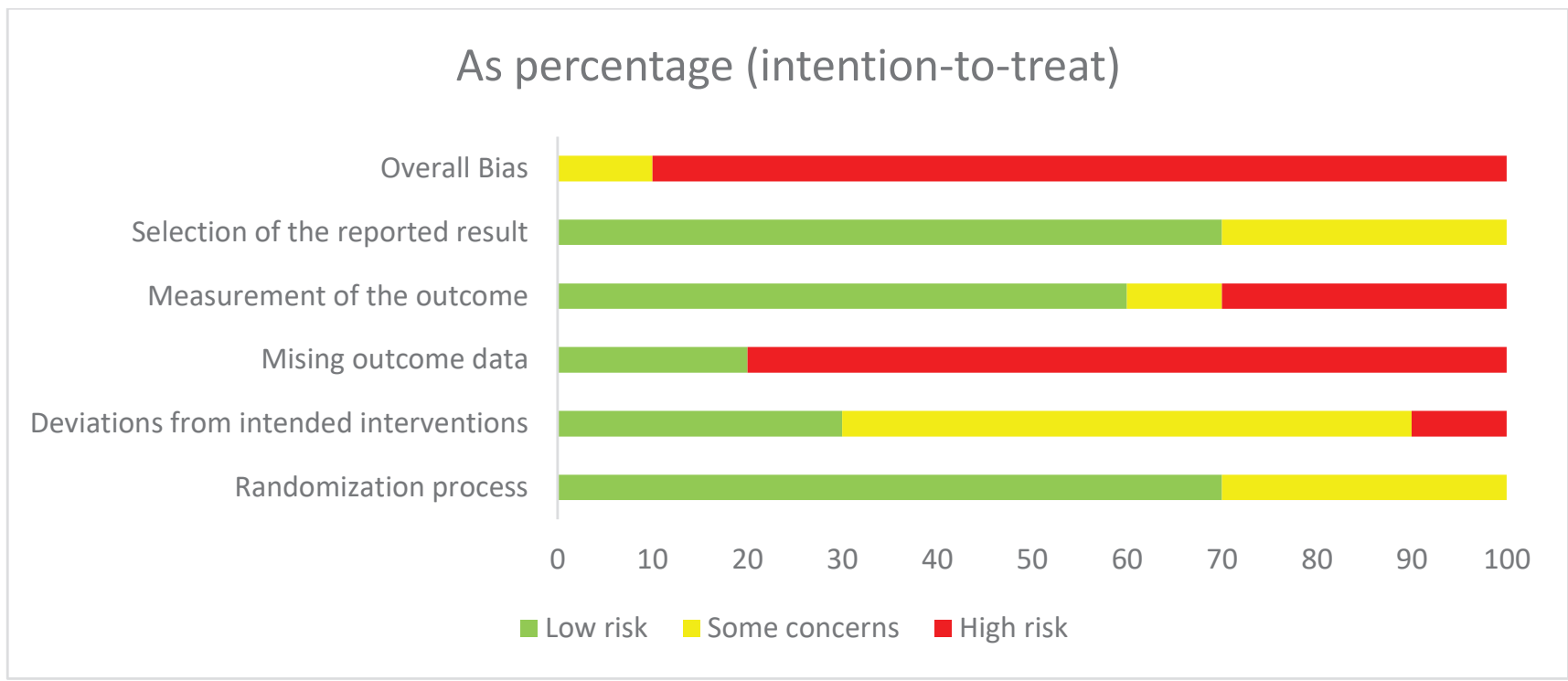

arm; the seventh trial did not have an NRT arm and the number lost-to-follow-up was highest in the no additional treatment arm. The TEC trial was the only included study that attempted to assess the impact of missing data in four sensitivity analyses ${ }^{45}$. The risk of bias assessment is summarized in Figure 1.

During the meta-analysis feasibility assessment for the smoking cessation endpoint, we found that the study populations of the identified RCTs were comparable for pooling. One possible outlier population was the Halpern et al. ${ }^{48} 2018$ trial, which may have involved light smokers (Supplementary file Appendix 3), and therefore a sensitivity analysis was conducted to assess the impact of this trial on the NMA results. The feasibility analysis also considered the main intervention, which was the type of ENDS and dosage of nicotine given to the participants. Only first- and second-generation ENDS were included. ENDS was the intervention in all trials, and it was compared with NRT, or ENNDS (no nicotine), or no additional treatment. The nicotine dose in the NRT arm was most commonly monotherapy and some studies included more moderate dosing such as 2 $\mathrm{mg}$ gum and $14 \mathrm{mg}$ patches (Table 2 ). The nicotine dose in ENDS arms ranged $5.4-18 \mathrm{mg} / \mathrm{mL}$ with the exception of the Lee ${ }^{49} 2019$ trial which reported a dose of $0.01 \mathrm{mg} / \mathrm{mL}$. A second sensitivity analysis was conducted excluding the trail of Lee ${ }^{49}$. Seven ${ }^{55}$ of the eight cessation RCTs were at high-risk of bias. All eight RCTs reported smoking cessation results at
24 or 26 weeks. Seven of the eight RCTs verified the self-reported data with biochemical analysis ${ }^{55}$, the study which did not use biochemical verification was excluded in the third sensitivity analysis.

\section{Cessation at 24 or 26 weeks}

Figure 2 presents the data and network relationship using the eight RCTs that measured smoking cessation at 24-26 weeks ${ }^{39,40,45,47-49,51,55}$.

\section{Figure 2. Evidence network for smoking cessation at} 24 or 26 weeks

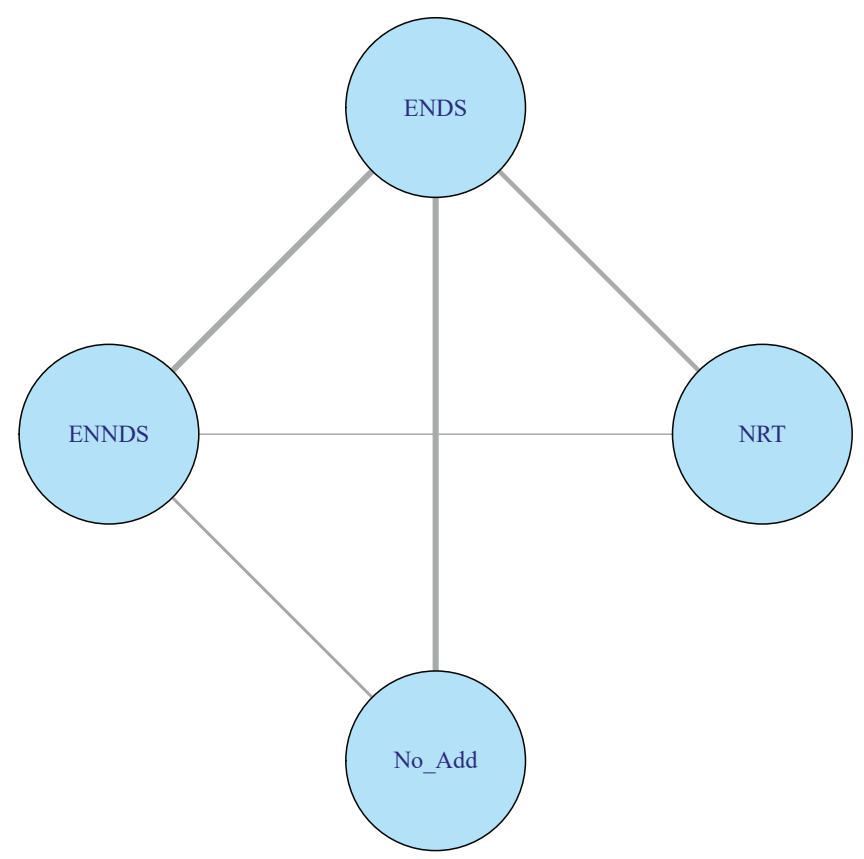


Figure 3. Network meta-analysis of smoking cessation at 24 or 26 weeks

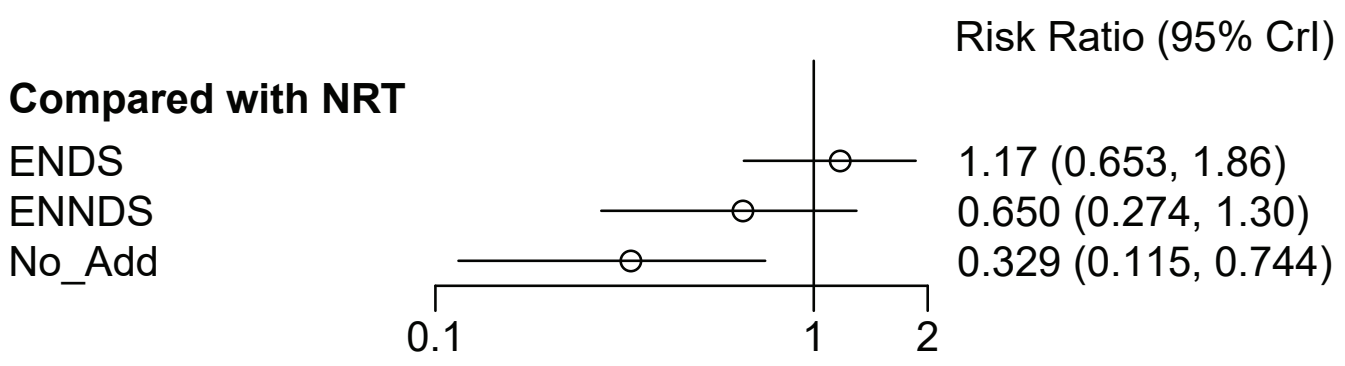

There was no statistically significant difference (evaluated using relative risk, RR, with 95\% credible interval, CrI) between the ENDS and the NRT groups ( $\mathrm{RR}=1.17$; 95\% CrI: 0.65-1.86) for smoking cessation at 24 or 26 weeks (Figure 3 ). There was no statistically significant difference between ENNDS (no nicotine) and NRT in achieving smoking cessation at 24 or 26 weeks ( $R R=0.65 ; 95 \%$ CrI: $0.27-1.30$ ). No additional treatment is less effective than NRT and this result is statistically significant $(\mathrm{RR}=0.33$; 95\% CrI: $0.12-$ 0.74 ). The meta-analysis feasibility assessment identified heterogeneity in ENDS type and nicotine dose and therefore a random-effects NMA model at 24-26 weeks was reported.

Three sensitivity analyses were carried out. The first excluded the RCT which appeared to include lighter smokers ${ }^{47}$, the second excluded the RCT which had not biochemically verified their cessation data at 24 weeks $^{45}$, and the third excluded the RCT with a lower dose of nicotine in the ENDS $\mathrm{arm}^{48}$. The results of these sensitivity analyses indicate that the main analysis was robust to assumptions relating to the smoking history of participants (NRT vs ENDS: $\mathrm{RR}=1.18$; 95\% CrI: $0.66-1.82$ vs $\mathrm{RR}=1.17$; 95\% CrI: 0.65-1.86) and inclusion of unverified data (ENDS vs NRT: $R R=0.93$; $95 \%$ CrI: $0.46-1.93$ vs $R R=1.17$; 95\% CrI: 0.65-1.86), and lower nicotine dose (NRT vs ENDS: $R R=1.35$; 95\% CrI: 0.73-2.34 vs RR=1.17; 95\% CrI: 0.65-1.86). There was no change to statistically significance in any sensitivity analysis, all continued to demonstrate no statistically significant difference between ENDS and NRT. The complete sensitivity analysis is presented in Supplementary file Appendix 8.

The consistency and inconsistency models produced very similar estimates of treatment effect, agreeing in terms of direction and magnitude of effect. The Deviance Information Criterion was marginally lower for the consistency model (27.2 vs 28.9), indicating that the consistency model was appropriate. There was no difference in the direction of effect, from the direct and indirect evidence, for any of the comparisons. There is no evidence of inconsistency in the treatment effects presented in NMA at 24-26 weeks.

\section{Cessation at 52 weeks}

We did not undertake an NMA of smoking cessation at 52 weeks due to limited data; only three RCTs reported data for this timepoint ${ }^{40,45,47}$. Each study compared ENDS to a different control, and RCT results were mixed. One study with 300 participants found that ENDS appears more effective than ENNDS (no nicotine) for smoking cessation ( $\mathrm{RR}=2.75 ; 95 \%$ CrI: 0.97-7.76), but the difference was not statistically significant ${ }^{40}$. One study showed that ENDS appears more effective than no additional treatment at 52 weeks ( $R R=6.11$; 95\% CrI: 0.33-113.24), but the difference was not statistically significant and the confidence intervals were very wide indicating a very small number of successful incidences of cessation ${ }^{47}$. One trial ( $\mathrm{N}=886$ participants) found that ENDS was more effective than NRT (RR=1.83; 95\% CrI: 1.30-2.58), and this difference was statistically significant ${ }^{45}$. The substantial uncertainty for all of these analyse $\mathrm{s}^{40,46,47}$ is attributable to the low number of successful events in each study coupled with the large numbers lost to follow-up (Supplementary file Appendix 6).

\section{Adverse events}

This systematic review found that standardized definitions were used to collect data on adverse events in two ${ }^{39,45}$ of the ten included RCTs. Only one of these specified the coding guidelines, Medical Dictionary for Regulatory Activities (MedDRA), that were used to 
classify the adverse events ${ }^{45}$. The adverse events in this study were all collected over a short period of time $(\leq 12$ months). A meta-analysis was not carried out as adverse events were not consistently reported, or classified.

European Medicines Agency classified adverse events were reported in nine RCTs $39,40,45,48,49,51-54$ and were recorded at 8 months ${ }^{52,53}, 12$ months $^{48,54}, 6$ months $\mathrm{s}^{39,49,51,54}$, or 52 weeks $^{40,45}$.

Documented adverse events included vital signs ${ }^{40,49,52}$ in three of the RCTs, and indicators of cardiovascular ${ }^{45,49,53}$ or psychiatric events ${ }^{40,45,49,54}$ in four of the ten RCTs (Supplementary file Appendix 9 ). Respiratory events (including shortness of breath and cough) $)^{40,45,48,49,52-54}$ and addiction associated potential and withdrawal events ${ }^{39,40,45,48,49,51}$ were documented in six of the ten RCTs each (Supplementary file Appendix 9). For all reported adverse events, the incidence of documented adverse events was lower in the control arms [NRT or ENNDS (no nicotine)] than in the ENDS arm, except for shortness of breath in two studies (highest in the ENNDS arm) and cough in one study (highest in NRT arm) (Supplementary file Appendix 9).

\section{Level and quality of evidence}

The authors assigned a Level-2 evidence rating using the Centre for Evidence-Based Medicine levels of evidence guidelines ${ }^{56}$ as we had eight RCTs in the NMA, but seven of the eight trials had a high risk of bias. With respect to the certainty of evidence ${ }^{57,58}$, there is low certainty of evidence that e-cigarettes or ENDS have the same levels of success in achieving smoking cessation as the regulated gold standard nicotine replacement therapies for cessation ${ }^{25}$. The low certainty is based on the results of the NMA at 6 months, because of the high-risk of bias in seven of the eight trials, the low number of successful events in the trials, and the high dropout rates.

There is a very low certainty of evidence or inconclusive evidence that ENDS have the same levels of or more success in achieving smoking cessation as other medically approved cessation interventions, based on three trials all with different comparators, for smoking cessation at 52 weeks.

\section{DISCUSSION}

The incidences of smoking cessation at 24-26 weeks, derived from this NMA of eight trials, were similar between ENDS and NRT groups $(\mathrm{RR}=1.17 ; 95 \%$ CrI: 0.65-1.86; low certainty of evidence). Three sensitivity analyses indicate that the main findings for 24-26 weeks were robust to assumptions relating to the smoking history of participants and inclusion of unverified data, and to the assumption of equivalence in nicotine dose. An NMA of smoking cessation at 52 weeks was not undertaken due to very limited data, and a narrative synthesis of evidence for effectiveness of e-cigarettes in smoking cessation at this time showed substantial uncertainly in individual RCT results. No serious adverse events were designated as being related to treatment in the six studies that intended to document serious adverse events; however, the procedure for determining if a serious adverse event was related to the smoking cessation intervention was often unclear. In general, safety and health impacts of e-cigarettes beyond 12 months are not yet established.

The complex and emergent nature of e-cigarettes presents a key challenge for public health policy makers and healthcare professionals, so it is critical that evidence synthesis is independent, transparent, makes best use of available and up-to-date evidence so as to usefully inform critical population and individual level decisions.

\section{Strengths and limitations}

There are a number of strengths to this systematic review. First, the systematic review and metaanalysis was conducted by a group who were unaligned to the conduct of the primary studies providing an independent assessment of evidence. Second, a comprehensive, robust and reproducible search strategy was used to capture all relevant trials. Third, different endpoints across studies have been disentangled and reported separated for accuracy. Finally, NMA, augmented with wellreasoned sensitivity analyses based on feasibility and appropriateness assessment, was chosen as the preferred method to fully analyze the available evidence base and optimizes precision; such analyses can deal with multi-arm trials.

A number of limitations in the primary studies restricted the extent of our evidence synthesis and our certainty in the findings. In general, the quality of the primary studies synthesized in this review was low, with all but one study assessed as being at high-risk of bias. Many studies were small, 
with high loss-to-follow-up and low numbers of participants achieving smoking abstinence, even at the proximal 24-26 weeks endpoint. Significant concerns regarding comparators and quality prevented synthesis of the 52 -week endpoint. While loss-to-follow-up is a common limitation in trials of smoking cessation interventions and procedures have been agreed for handling outcomes, this source of information error was different in the included trials, with loss-to-follow-up generally greater in NRT treated trial arms, which underscores the importance of achieving both high and equal response rates across compared groups to limit bias $^{59}$. This dependence on the quality of primary research is a well-recognized inherent limitation of evidence synthesis, and efforts have been made in this review to address this challenge through transparent reporting ${ }^{60}$, disentanglement of endpoints, and sensitivity analyses. E-cigarettes are not a standardized intervention. A variety of firstand second-generation e-cigarettes were tested, and the nicotine doses varied, although the impact of dose was found to be robust in sensitivity analysis. NRT dosing in the comparator arm was commonly monotherapy and sometimes includes less intense dosing. While monotherapy and lower dosage may replicate how some people use NRT in a selfmanaged cessation attempt, it is generally at odds with well-established evidence and clinical practice in treating tobacco addiction where best practice advice on NRT is combination therapy at more intense and more optimal nicotine dosages ${ }^{18}$. These issues with both the active and comparison arms of the studies present challenges to generalizability.

A limitation of our NMA is that we did not include studies that reported data for NRT versus control treatments alone. The reason for this was twofold, firstly our primary focus was on the efficacy of e-cigarettes versus standard care for smoking cessation, the efficacy of NRT for this purpose has already been proven ${ }^{61}$. Secondly, this research team did not have the resources required to expand the search to include data for this comparison, it would have at least tripled the workload and meant that the research team would not have been able to address the primary research question. Future research could consider an expanded network meta-analysis of all smoking cessation treatments, noting that this would be a resource intensive project.

This review concludes that e-cigarettes with nicotine may be as effective as NRT in achieving smoking cessation at 24-26 weeks (eight RCTs). Previous reviews in this important area have been mixed. Rahman et al. ${ }^{23}$ pooled data from two trials and found that ENDS, compared with ENNDS (no nicotine), helped smokers to stop smoking long-term ${ }^{23}$. On the other hand, a 2016 review by Kalkhoran and Glatzlost ${ }^{20}$ concluded that e-cigarettes are associated with significantly lower quit rates among smokers ${ }^{20}$. More recent reviews by El Dib et al. ${ }^{17}$ and Khoudigian et al. ${ }^{19}$, based on two and three trials, respectively, report findings similar to ours - that the incidences of smoking cessation at 24 or 26 weeks for ENDS versus ENNDS (no nicotine) indicate that an e-cigarette with nicotine is marginally better than one without nicotine, but that this result is not statistically significantly and there is a high-level of uncertainty. A systematic review by Malas et al. ${ }^{21}$ in 2016 , elected not to conduct a meta-analysis because of the heterogeneity of the data. They concluded that, while the majority of included studies (mostly non-RCTs) suggested a positive relationship between ENDS and smoking cessation, the evidence remained inconclusive due to the low quality of the published data. According to a 2020 World Health Organization report, based mostly on the US Academies of Sciences systematic review, some types of ENDS aid in smoking cessation in certain circumstances, but the evidence is insufficient to issue a general recommendation to use any type of e-cigarette (nicotine or non-nicotine) as a cessation aid for all smokers ${ }^{62}$.

Wang et al. ${ }^{24}$ published a systematic review and meta-analysis in 2021, looking at both observational and RCT data. Observational and RCT data were analyzed separately, with the observational indicating that ENDS were not associated with increased smoking cessation but the RCT analysis found that ENDS were associated with increased smoking cessation in a controlled situation.

A systematic review leading to NMA was also published in 2021 by Chan et al. ${ }^{22}$ who found that ENDS users were more likely to quit smoking than control or NRT users; however, they stipulated that more high quality studies are required to ascertain the true effect of ENDS on smoking cessation. 
Chan et al. ${ }^{22}$ made a number of assumptions in their analysis that were different to ours including: pooling of data over multiple timepoints and pooling of all non-nicotine treatment arms, i.e. assuming non-nicotine e-cigarettes are the same as usual care.

While this manuscript was being completed, a revision of a Cochrane review of e-cigarettes for smoking cessation ${ }^{18}$, which is now designated as a living review, was published. While there are similarities in the research question, there are a number of differences between this work and that presented in the Cochrane review. The most important of these relate to the type and timing of the outcomes that are summarized. In our current work, to be consistent with standards in the area of smoking cessation trials ${ }^{26}$, we have excluded studies where the outcome is not continuous abstinence without relapse throughout the follow-up period, e.g. Lee et al. ${ }^{54}$ which measured 7 -day point prevalence abstinence only. In addition, we have separately analyzed outcomes at 24 or 26 weeks and those reported at 52 weeks rather than take data at the longest follow-up only and present these to the systematic review user as the same endpoint. Finally, a key difference is the use of Network Meta-Analysis, which means a broader collection of indirect evidence (when compared to pairwise meta-analysis) is included in the synthesis. We consider our decisions clinically and methodologically sound. The Cochrane review reported that there is moderatecertainty evidence that ENDS increase quit rates compared to NRT. This conclusion is based on a pairwise meta-analysis of three RCTs of which two reported data at 6 months, one at 12 months and one did not conform to the strict definition of smoking abstinence. We report low and very low certainty of evidence in our results because of the high risk of bias in most of the included trials, the high numbers lost to follow-up in the trials, and the low success rates of all trial interventions. The final difference is that the review presented in this article employed the newer Cochrane Collaboration ROB2 tool while Hartman-Boyce et al. ${ }^{18}$ used the previous version 1 of this tool, which may explain differences in the two groups assessment of bias results.

\section{Future perspectives}

To better inform policy and clinical practice, future studies of e-cigarettes should be designed with comparator arms that offer participants intense and optimal NRT as well as other more effective smoking cessation interventions. The effectiveness of e-cigarettes at 24-26 and 52 weeks needs to be established as to date results are mixed and of low or very low quality. The question of whether e-cigarettes may have different effectiveness for different populations of smokers also needs to be addressed, since the trade-offs at individual patient level between the harms, benefits and uncertainties are different. Further long-term large-scale multi-country RCTs are needed to assess the efficacy and safety of electronic cigarettes (e-cigarettes) in helping people who smoke to achieve abstinence. If e-cigarettes are to be used as a treatment for smoking cessation, then they need to have an established long-term safety profile. This links with the questions of their regulation as medical or consumer products, which will be especially important for clinicians. Recommending e-cigarettes has been demonstrated to lead to their continuing use by people who are successful in stopping smoking tobacco cigarettes as well as by people who experience unsuccessful quit attempts and end up using both e-cigarettes and tobacco cigarettes (dual use) $)^{45,63}$. Some of these people may not have tried e-cigarettes in the absence of a therapeutic recommendation ${ }^{45,63}$. In a mapping exercise on the harms and benefits of e-cigarettes, McCarthy et al. ${ }^{65}$ noted that many studies showed that dual use of e-cigarettes and conventional tobacco cigarettes was not less harmful than smoking conventional tobacco cigarettes only, thereby raising questions about the smoking reduction benefit of e-cigarettes. Any future studies on e-cigarettes and smoking cessation need to monitor on-going use of e-cigarettes, in particular dual use, as a consequence of recommending e-cigarette use for smoking cessation. While reporting a finding of greater effectiveness of e-cigarettes compared with NRT with moderate certainty, the recent Cochrane review also identifies a need for more studies with higher quality to build a more reliable evidence-base.

The systematic review evidence on the efficacy of ENDS for smoking cessation to date shows contradictory results. There are, of course, explanations for these differences, however, it is abundantly clear that if research groups can use standard evidence synthesis techniques and generate 
different results, then further high-quality primary evidence is urgently required.

The scale of disease, disability and premature mortality caused by smoking continues to demand an urgent response $\mathrm{e}^{64}$, which not only includes stronger initiation prevention, but which also provides more effective cessation support to help save the lives of people who currently smoke. Policy-makers and healthcare professionals alike are struggling to navigate and resolve the often divisive debate ${ }^{65}$ on what, if any, role e-cigarettes may play in this context ${ }^{6-8}$. Both sets of decision-makers face tradeoffs between harms, benefits and uncertainties at the individual level, however, policy-makers face the added consideration of potential trade-offs across population groups and between short- and long-term goals for public health.

\section{CONCLUSIONS}

Much needed progress is being made towards providing robust and precise scientific evidence to enable clinical and policy decisions about e-cigarettes to be made with greater confidence ${ }^{12}$. However, the fact that this review and recent Cochrane review have arrived at different conclusions, largely through differences in assumptions around the data included and the overall method of analysis, while both following accepted evidence-synthesis practice, will add to concerns about the role of systematic reviews in supporting decision-making ${ }^{66,67}$. This should not be the case, however. Both reviews are aligned on the need for further well-designed, adequately powered, and carefully conducted primary studies with clearly reported cessation and safety outcomes to support clinical and policy decision-making. Overall, these reviews indicate that the research question 'should ENDS be recommended to aid smoking cessation?' is far from answered. Further research should be synthesized by review teams that are independent of the primary trial groups to avoid investigator bias to increase transparency and trust in findings.

However, urgent action to tackle the harms of smoking based on what we know is safe and works cannot and should not await resolving questions about e-cigarettes. Healthcare professionals must work to close the implementation gap between existing knowledge on smoking cessation interventions with well-established effectiveness and safety profiles and the reality of care, as it is too often experienced by people who smoke who commonly miss the opportunity to receive clinical advice and support at a time when it could have greatest impact ${ }^{68-70}$. Policy-makers should act now to ensure that they have fully protected children and young people from the harms of e-cigarettes and the potential to undermine progress in tobacco control through facilitating smoking initiation ${ }^{71}$. While determining whether the risk-benefit profile of e-cigarettes in some population groups is best mobilized through their regulation as consumer products or through the same regulation as other licensed medicines ${ }^{72}$, there is much more policymakers can do to immediately support healthcare professionals and patients to maximize the uptake of existing, well-regulated smoking cessation interventions ${ }^{73-75}$.

The e-cigarette debate continues to predominate tobacco control internationally. Do we need to await a final verdict on a single potential game-changer to take action now to change the tobacco control game and bring it to an end ${ }^{76}$ ? While our study indicates that research evidence is not yet sufficient in volume or quality to conclude the e-cigarette debate, it affirms that clinician and policy-makers urgency to tackle the harms of smoking can be focused with confidence on augmenting support for interventions with well-proven safety and effectiveness.

\section{REFERENCES}

1. Hsu G, Sun JY, Zhu SH. Evolution of Electronic Cigarette Brands From 2013-2014 to 2016-2017: Analysis of Brand Websites. J Med Internet Res. 2018;20(3):e80. doi:10.2196/jmir.8550

2. Benmarhnia T, Pierce John P, Leas E, et al. Can E-Cigarettes and Pharmaceutical Aids Increase Smoking Cessation and Reduce Cigarette Consumption? Findings From a Nationally Representative Cohort of American Smokers. American journal of epidemiology. 2018;187(11):2397-2404. doi:10.1093/aje/kwy129

3. Dawkins LE, Kimber CF, Doig M, Feyerabend C, Corcoran O. Self-titration by experienced e-cigarette users: blood nicotine delivery and subjective effects. Psychopharmacology. 2016;233(15-16):2933-2941. doi:0.1007/s00213-016-4338-2

4. Dawkins L, Cox S, Goniewicz M, et al. 'Real-world' compensatory behaviour with low nicotine concentration e-liquid: subjective effects and nicotine, acrolein and formaldehyde exposure. Addiction. 2018;113(10):18741882. doi:10.1111/add.14271 
5. Soar K, Kimber C, McRobbie H, Dawkins LE. Nicotine absorption from e-cigarettes over 12 months. Addict Behav. 2019;91:102-105. doi:10.1016/j.addbeh.2018.07.019

6. Stimson GV, Thom B, Costall P. Disruptive innovations: the rise of the electronic cigarette. Int J Drug Policy. 2014;25(4):653-655. doi:10.1016/j.drugpo.2014.05.003

7. Abrams DB. Promise and peril of e-cigarettes: can disruptive technology make cigarettes obsolete? JAMA. 2014;311(2):135-136. doi:10.1001/jama.2013.285347

8. Fagerstrom K, Etter JF, Unger JB. E-cigarettes: a disruptive technology that revolutionizes our field? Nicotine Tob Res. 2015;17(2):125-126. doi:10.1093/ntr/ntu240

9. Green LW, Fielding JE, Brownson RC. The Debate About Electronic Cigarettes: Harm Minimization or the Precautionary Principle. Annu Rev Public Health. 2018;39:189-191. doi:10.1146/annurev-publhealth-102417-124810

10. Kennedy RD, Awopegba A, De León E, Gohen JE. Global approaches to regulating electronic cigarettes. Tob Control. 2017;26(4):440-445. doi:10.1136/tobaccocontrol-2016-053179

11. Erku DA, Gartner CE, Morphett K, Steadman KJ. Beliefs and Self-reported Practices of Health Care Professionals Regarding Electronic Nicotine Delivery Systems: A MixedMethods Systematic Review and Synthesis. Nicotine Tob Res. 2020;22(5):619-629. doi:10.1093/ntr/ntz046

12. Newton JN, Dockrell M, Marczylo T. Making sense of the latest evidence on electronic cigarettes. Lancet. 2018;391(10121):639-642. doi:10.1016/S0140-6736(18)30202-2

13. The debate over e-cigarettes demands stronger evidence of their value. Nature. 2019;570(7762):415. doi:10.1038/d41586-019-01785-4

14. Zhang Y, Akl EA, Schünemann HJ. Using systematic reviews in guideline development: the GRADE approach. Res Synth Methods. 2018. doi:10.1002/jrsm.1313

15. Greenhalgh T, Malterud K. Systematic Reviews for Policymaking: Muddling Through. Am J Public Health. 2017;107(1):97-99. doi:10.2105/AJPH.2016.303557

16. Fairchild AL, Bayer R. Public health. Smoke and fire over e-cigarettes. Science. 2015;347(6220):375-376. doi:10.1126/science. 1260761

17. El Dib R, Suzumura EA, Akl EA, et al. Electronic nicotine delivery systems and/or electronic non-nicotine delivery systems for tobacco smoking cessation or reduction: a systematic review and meta-analysis. BMJ Open. 2017;7(2):e012680. doi:10.1136/bmjopen-2016-012680

18. Hartmann-Boyce J, McRobbie H, Lindson N, et al. Electronic cigarettes for smoking cessation. Cochrane Database of Systematic Reviews. 2020;10(10):CD010216. doi:10.1002/14651858.CD010216.pub4

19. Khoudigian S, Devji T, Lytvyn L, Campbell K, Hopkins R, O'Reilly D. The efficacy and short-term effects of electronic cigarettes as a method for smoking cessation: a systematic review and a meta-analysis. Int J Public Health.
2016;61(2):257-267. doi:10.1007/s00038-016-0786-z

20. Kalkhoran S, Glantz SA. E-cigarettes and smoking cessation in real-world and clinical settings: a systematic review and meta-analysis. Lancet Respir Med. 2016;4(2):116-128. doi:10.1016/S2213-2600(15)00521-4

21. Malas M, van der Tempel J, Schwartz R, et al. Electronic cigarettes for smoking cessation: a systematic review. Nicotine Tob Res. 2016;18(10):1926-1936. doi:10.1093/ntr/ntw119

22. Chan GCK, Stjepanović D, Lim C, et al. A systematic review of randomized controlled trials and network meta-analysis of e-cigarettes for smoking cessation. Addict Behav. 2021;119:106912. doi:10.1016/j.addbeh.2021.106912

23. Rahman MA, Hann N, Wilson A, Mnatzaganian G, Worrall-Carter L. E-cigarettes and smoking cessation: evidence from a systematic review and meta-analysis. PloS one. 2015;10(3):e0122544. doi:10.1371/journal.pone.0122544

24. Wang RJ, Bhadriraju S, Glantz SA. E-Cigarette Use and Adult Cigarette Smoking Cessation: A MetaAnalysis. Am J Public Health. 2021;111(2):230-246. doi:10.2105/AJPH.2020.305999

25. European Medicines Agency. Guideline on the development of medicinal products for the treatment of smoking. European Medicines Agency; 2018. Accessed May 31, 2021. https://www.ema.europa.eu/en/ documents/scientific-guideline/guideline-developmentmedicinal-products-treatment-smoking_en.pdf

26. West R, Hajek P, Stead L, Stapleton J. Outcome criteria in smoking cessation trials: proposal for a common standard. Addiction. 2005;100(3):299-303. doi:10.1111/j.1360-0443.2004.00995.x

27. Cheung KL, de Ruijter D, Hiligsmann M, et al. Exploring consensus on how to measure smoking cessation. A Delphi study. BMC Public Health. 2017;17(1):890. doi:10.1186/s12889-017-4902-7

28. Sterne JAC, Savović J, Page MJ, et al. RoB 2: A revised Cochrane risk-of-bias tool for randomized trials. BMJ. 2019;366:14898. Revised August 18, 2019. Accessed May 31, 2021. https://sites.google.com/site/riskofbiastool/ welcome/rob-2-0-tool?authuser $=0$

29. Quigley JM, Woods B, Thompson J, Bryden PA, Scott DA. PRM209 - Confronting heterogeneity: Using systematic review effectively for meta-analysis. Value Health. 2013;16(7):A612. doi:10.1016/j.jval.2013.08.1763

30. Cope S, Zhang J, Saletan S, Smiechowski B, Jansen JP, Schmid P. A process for assessing the feasibility of a network meta-analysis: a case study of everolimus in combination with hormonal therapy versus chemotherapy for advanced breast cancer. BMC Med. 2014;12:93. doi:10.1186/1741-7015-12-93

31. Nilan K, McNeill A, Murray RL, McKeever TM, Raw M. A survey of tobacco dependence treatment guidelines content in 61 countries. Addiction. 2018;113(8):1499- 
1506. doi:10.1111/add.14204

32. Cahill K, Stevens S, Perera R, Lancaster T. Pharmacological interventions for smoking cessation: an overview and network meta-analysis. Cochrane Database Syst Rev. 2013(5):Cd009329. doi:10.1002/14651858.CD009329.pub2

33. Salanti G. Indirect and mixed-treatment comparison, network, or multiple-treatments meta-analysis: many names, many benefits, many concerns for the next generation evidence synthesis tool. Res Synth Methods. 2012;3(2):80-97. doi:10.1002/jrsm.1037

34. Chaimani A, Caldwell DM, Li T, Higgins JPT, Salanti G. Chapter 11: Undertaking network meta-analyses. In: Higgins JPT, Thomas J, eds. Cochrane Handbook for Systematic Reviews; 2019. v. 6.2. Updated 2021. Accessed May 31, 2021. https://training.cochrane.org/ handbook/current

35. gemtc: Network Meta-Analysis Using Bayesian Methods. Version 1.0-1. Accessed May 31, 2021. https://cran.rproject.org/web/packages/gemtc/index.html

36. R: A language and environment for statistical computing. R Foundation for Statistical Computing; 2017. Accessed May 32, 2021. https://www.R-project.org/

37. Dias S, Ades AE, Welton NJ, Jansen JP, Sutton AJ. Network meta-analysis for decision making. John Wiley \& Sons Ltd.; 2018.

38. RDocumentation. meta-package: meta: Brief overview of methods and general hints. meta version 4.9-7. Published 2019. Accessed May 31, 2021. https://www. rdocumentation.org/packages/meta/versions/4.9-7/ topics/meta-package

39. Schwarzer G, Carpenter J, Rücker G. Meta-analysis with R. Institut fur Medizinische Biometrie und Statistik (IMBI); 2019. Accessed 2020. http://www.imbi.unifreiburg.de/lehre/lehrbuecher/meta-analysis-with-r

40. Bullen C, Howe C, Laugesen M, et al. Electronic cigarettes for smoking cessation: A randomised controlled trial. Lancet. 2013;382(9905):1629-1637. doi:10.1016/S0140-6736(13)61842-5

41. Caponnetto P, Campagna D, Cibella F, et al. EffiCiency and Safety of an eLectronic cigAreTte (ECLAT) as tobacco cigarettes substitute: a prospective 12-month randomized control design study. PloS one. 2013;8(6):e66317. doi:10.1371/journal.pone.0066317

42. Campagna D, Cibella F, Caponnetto P, et al. Changes in breathomics from a 1-year randomized smoking cessation trial of electronic cigarettes. Eur J Clin Invest. 2016;46(8):698-706. doi:10.1111/eci.12651

43. Cibella F, Campagna D, Caponnetto P, et al. Lung function and respiratory symptoms in a randomized smoking cessation trial of electronic cigarettes. Clin Sci. 2016;130(21):1929-1937. doi:10.1042/CS20160268

44. Farsalinos K, Cibella F, Caponnetto P, et al. Effect of continuous smoking reduction and abstinence on blood pressure and heart rate in smokers switching to electronic cigarettes. Intern Emerg Med. 2016;11(1):85-
94. doi:10.1007/s11739-015-1361-y

45. Russo C, Cibella F, Caponnetto P, et al. Evaluation of Post Cessation Weight Gain in a 1-Year Randomized Smoking Cessation Trial of Electronic Cigarettes. Sci Rep. 2016;6:18763. doi:10.1038/srep18763

46. Hajek P, Phillips-Waller A, Przulj D, et al. A randomized trial of e-cigarettes versus nicotine-replacement therapy. N Engl J Med. 2019a;380(7):629-637. doi:10.1056/NEJMoa1808779

47. Hajek P, Phillips-Waller A, Przulj D, et al. E-cigarettes compared with nicotine replacement therapy within the UK Stop Smoking Services: the TEC RCT. Health Technol Assess. 2019;23(43):1-82. doi:10.3310/hta23430

48. Halpern SD, Harhay MO, Saulsgiver K, Brophy C, Troxel $\mathrm{AB}$, Volpp KG. A pragmatic trial of e-cigarettes, incentives, and drugs for smoking cessation. N Engl J Med. 2018;378(24):2302-2310. doi:10.1056/NEJMsa1715757

49. Lee SH, Ahn SH, Cheong YS. Effect of electronic cigarettes on smoking reduction and cessation in Korean male smokers: A randomized controlled study. J Am Board Fam Med. 2019;32(4):567-574. doi:10.3122/jabfm.2019.04.180384

50. Masiero M, Lucchiari C, Mazzocco K, et al. E-cigarettes may support smokers with high smoking-related risk awareness to stop smoking in the short run: Preliminary results by randomized controlled trial. Nicotine Tob Res. 2019;21(1):119-126. doi:10.1093/ntr/nty047

51. Lucchiari C, Masiero M, Mazzocco K, et al. Benefits of e-cigarettes in smoking reduction and in pulmonary health among chronic smokers undergoing a lung cancer screening program at 6 months. Addict Behav. 2020;103:106222. doi:10.1016/j.addbeh.2019.106222

52. Holliday R, Preshaw PM, Ryan V, et al. A feasibility study with embedded pilot randomised controlled trial and process evaluation of electronic cigarettes for smoking cessation in patients with periodontitis. Pilot Feasibility Stud. 2019;5:74. doi:10.1186/s40814-019-0451-4

53. Hatsukami D, Meier E, Lindgren BR, et al. A randomized clinical trial examining the effects of instructions for electronic cigarette use on smoking-related behaviors, and biomarkers of exposure. Nicotine Tob Res. 22(9):15241532. doi:10.1093/ntr/ntz233

54. Lee SM, Tenney R, Wallace AW, Arjomandi M. E-cigarettes versus nicotine patches for perioperative smoking cessation: a pilot randomized trial. PeerJ. 2018;6:e5609. doi:10.7717/peerj.5609

55. Eisenberg MJ, Hébert-Losier A, Windle SB, et al. Effect of e-Cigarettes Plus Counseling vs Counseling Alone on Smoking Cessation: A Randomized Clinical Trial. JAMA. 2020;324(18):1844-1854. doi:10.1001/jama.2020.18889

56. Benowitz NL, Bernert JT, Foulds J, et al. Biochemical Verification of Tobacco Use and Abstinence: 2019 Update. Nicotine Tob Res. 2020;22(7):1086-1097. doi:10.1093/ntr/ntz132

57. Centre for Evidence-Based Medicine. Oxford Centre for 
Evidence-Based Medicine - Levels of Evidence (March 2009). Published 2009. Accessed May 31, 2021. https:// www.cebm.net/2009/06/oxford-centre-evidence-basedmedicine-levels-evidence-march-2009/

58. Schünemann He, Brożek Je, Guyatt Ge, Oxman Ae, eds. GRADE handbook. Updated October 2013. Accessed May 31, 2021. https://gdt.gradepro.org/app/handbook/ handbook.html\#h.u1371quprl84

59. Schünemann HJ, Higgins JPT, Vist GE, Glasziou P, Akl EA, Skoetz N, Guyatt GH. Chapter 14: Completing 'Summary of findings' tables and grading the certainty of the evidence. In: Higgins JPT, Thomas J, Chandler J, Cumpston M, Li T, Page MJ, Welch VA, eds. Cochrane Handbook for Systematic Reviews of Interventions. Version 6.2. Updated February 2021. Accessed May 31, 2021. www.training.cochrane.org/handbook

60. Twardella D, Brenner H. Implications of Nonresponse Patterns in the Analysis of Smoking Cessation Trials. Nicotine Tob Res. 2008;10(5):891-896. doi:10.1080/14622200802027149

61. Egger M, Smith GD, Sterne JA. Uses and abuses of meta-analysis. Clin Med. 2001;1(6):478-484. doi:10.7861/clinmedicine.1-6-478

62. Health Information and Quality Authority. Health technology assessment (HTA) of smoking cessation interventions. Health Information and Quality Authority; 2017. Updated April 10, 2017. Accessed May 31, 2021. https://www.hiqa.ie/sites/default/files/2017-04/ Smoking\%20Cessation\%20HTA.pdf. Published 2017

63. World Health Organization. Electronic nicotine and nonnicotine delivery systems: a brief. WHO Regional Office for Europe; 2020. Accessed May 31, 2021. https://www. euro.who.int/__data/assets/pdf_file/0009/443673/ Electronic-nicotine-and-non-nicotine-delivery-systemsbrief-eng.pdf

64. Stein JH, Korcarz CE. E-Cigarettes versus NicotineReplacement Therapy for Smoking Cessation. N Engl J Med. 2019;380(20):1973-1974. doi:10.1111/add.15628

65. McCarthy A, Lee C, Long J. Harms and benefits of e-cigarettes and heat-not-burn tobacco products: A literature map. Health Research Board; 2020. Accessed May 31, 2021. https://www.hrb.ie/ fileadmin/2._Plugin_related_files/Publications/2020_ publication-related_files/2020_HIE/Evidence_Centre/ Harms_and_benefits_of_e-cigarettes_and_heat-notburn_tobacco_products_Literature_map.pdf

66. Reitsma MB, Fullman N, Ng M, et al. Smoking prevalence and attributable disease burden in 195 countries and territories, 1990-2015: a systematic analysis from the Global Burden of Disease Study 2015. The Lancet. 2017;389(10082):18851906. doi:10.1016/S0140-6736(17)30819-X

67. Malone RE. The Race to a Tobacco Endgame. Tob Control. 2016;25(6):607-608. doi:10.1136/tobaccocontrol-2016-053466

68. Fox DM. Evidence and Health Policy: Using and Regulating Systematic Reviews. Am J Public Health.
2017;107(1):88-92. doi:10.2105/AJPH.2016.303485

69. Bero L. Systematic Review: A Method at Risk for Being Corrupted. Am J Public Health. 2017;107(1):93-96. doi:10.2105/AJPH.2016.303518

70. Fiore MC, Goplerud E, Schroeder SA. The Joint Commission's new tobacco-cessation measureswill hospitals do the right thing? N Engl J Med. 2012;366(13):1172-1174. doi:10.1056/NEJMp1115176

71. Pack QR, Priya A, Lagu TC, Pekow PS, Rigotti NA, Lindenauer PK. Smoking cessation pharmacotherapy among smokers hospitalized for coronary heart disease. JAMA Internal Medicine. 2017;177(10):1525-1527. doi:10.1001/jamainternmed.2017.3489

72. Bogdanovica I, Agrawal S, Gregory B, et al. What is the quality of smoking cessation advice in guidelines of tobacco-related diseases? Clin Med. 2015;15(6):546-549. doi:10.7861/clinmedicine.15-6-546

73. O'Brien D, Long J, Quigley J, Lee C, McCarthy A, Kavanagh P. Association between electronic cigarette use and tobacco cigarette smoking initiation in adolescents: A systematic review and meta-analysis. BMC Public Health. 2021;21:954. doi:10.1186/s12889-021-10935-1

74. Gornall J. Electronic cigarettes: medical device or consumer product? BMJ. 2012;345:e6417. doi:10.1136/bmj.e6417

75. Huang J, Gwarnicki C, Xu X, Caraballo RS, Wada R, Chaloupka FJ. A comprehensive examination of own- and cross-price elasticities of tobacco and nicotine replacement products in the U.S. Prev Med. 2018;117:107-114. doi:10.1016/j.ypmed.2018.04.024

76. Boyle R, Solberg L, Fiore M. Use of electronic health records to support smoking cessation. Cochrane Database Syst Rev. 2014;2014(12):Cd008743. doi:10.1002/14651858.CD008743.pub3

77. van den Brand FA, Nagelhout GE, Reda AA, et al. Healthcare financing systems for increasing the use of tobacco dependence treatment. Cochrane Database Syst Rev. 2017;9(9):Cd004305. doi:10.1002/14651858.CD004305.pub5

78. McDaniel PA, Smith EA, Malone RE. The tobacco endgame: a qualitative review and synthesis. Tob Control. 2016;25(5):594604. doi:10.1136/tobaccocontrol-2015-052356 
CONFLICTS OF INTEREST

The authors have each completed and submitted an ICMJE Form for Disclosure of Potential Conflicts of Interest. The authors declare that they have no competing interests, financial or otherwise, related to the current work. P. Kavanagh reports unpaid role as Chair Guideline Development at Group-National Stop Smoking, the National Stop Smoking Guidelines reference HRB project upon which this paper was based on (https:// www.hrb.ie/publications/publication/electronic-cigarette-and-smokingcessation-an-evidence-review/).

\section{FUNDING}

This article was funded as part of the routine public service work of the Health Research Board, Ireland.

\section{ETHICAL APPROVAL AND INFORMED CONSENT}

Ethical approval and informed consent were not required for this study.

\section{DATA AVAILABILITY}

Data sharing is not applicable to this article as no new data were created.

\section{AUTHORS' CONTRIBUTIONS}

JQ screened abstracts and full text, validated extracted data, carried out part of the risk of bias analysis, and did the statistical analysis. CW validated the outputs of the analysis and provided input on the draft report. CL carried out the searches, screened abstracts, and provided comment on the manuscript draft. JL was involved in project conception, extracted the data, carried out part the risk of bias analysis, and provided comment on the manuscript draft. HK did full text screening, extracted the data, carried out part of the risk of bias analysis, and provided comment on the manuscript draft. AMcC was involved in project conception, screened abstracts, and provided comment on the manuscript draft. PK was involved in project conception and assisted in developing the first draft of the manuscript providing public health policy and clinical input.

\section{PROVENANCE AND PEER REVIEW}

Not commissioned; externally peer reviewed. 